\title{
STUDY ON AUTHORIZATION MANAGEMENT MODEL OF ONE-STOP OFFICE SYSTEM
}

\author{
Jianwei Yue $^{1,2}$, Hongchun Cai ${ }^{1, *}$, Luyao Chen ${ }^{1}$, Wei Zhuang ${ }^{1}$ \\ ${ }^{1}$ College of Resources Science and Technology, Beijing Normal University, Beijing, China, \\ 00875 \\ ${ }^{2}$ Academy of Disaster Reduction and Emergency Management Ministry of Civil Affairs \& \\ Ministry of Education, Beijing Normal University, Beijing, China, 100875 \\ * Corresponding author, Address: College of Resources Science and Technology, Beijing \\ Normal University, No. 19 XinJie KouWai Street, Beijing, 100875 P. R. China, Tel: +86- \\ 10-58807713, Fax: +86-10-58806173,Email: Caihc@lgy.cn
}

Abstract: An authorization management model named five-layer-two-section is proposed through studying on problems of authorization management model and role network model. The model inherits the thought of role network model, and at the same time it expands the role network model by putting website into management model, so as to more accurate authority management. And it puts user and post into one system, and role, activity, website into another. The model, combining rough regulation and meticulous adjustment, integrity and flexibility, could greatly decrease the complexity of the authorization management and the probability of making mistake.

Keywords: authorization management, one-stop office system, RBAC

\section{INTRODUCTION}

Information technology is a key force for social development and it is very necessary for modern society to enhance informatization, a key factor to promote economic development and new countryside construction. Large scale application of information technology is becoming an important technological method to enhance the modernization of countryside and to develop its economy. At present, office systems have been built in governmental management department of all levels. But these systems are mutually independent, which results to some inconvenience. Thus, one of the urgent problems is to integrate these separated systems to set up a one-stop

Yue, J., Cai, H., Chen, L. and Zhuang, W., 2008, in IFIP International Federation for Information Processing, Volume 259; Computer and Computing Technologies in Agriculture, Vol. 2; Daoliang Li; (Boston: Springer), pp. 925-931. 
office system which could enable unified access to different systems and make system maintenance more efficiently. Because of the complicated relations among office systems which hold different functions, there are numerous problems when the one-stop system is being built by integrating several systems together. One of these problems is authority management (Ye Xin et al. 2006).

Fig. 1(a) shows common authorization management model, which can be accepted as Role-based Access Control (RBAC) developed by Sandhu (Yan han et al. 2006, Li Huaiming et al. 2006, Han Shengju et al. 2006). This model separates users and specific transaction process, and combines the two with role. In this model, the change of user's roles can meet the requirement of user authority change, which appears efficient and simple. However, this efficiency and complexity are just limited to the number of user's roles. If a user has many roles, the operation of change user's authority must be very complicated. This would especially be true in the following example. There are several systems in a certain department, and the user's authority would be changed a lot when an employee was promoted to a manager. Accordingly, his authority may be confined in one system before, but after promoting his authority would expand to all the systems that the manager can $\log$ in. This kind of user authority change in the system is convoluted and complicated.

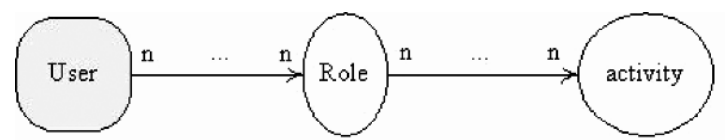

(a) $\mathrm{RBAC}$ authority management model

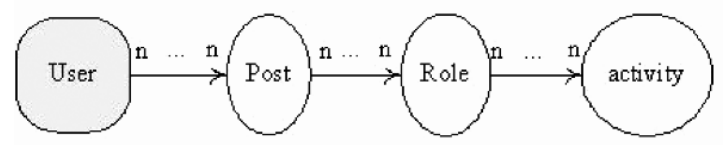

(b) RNM authority management model

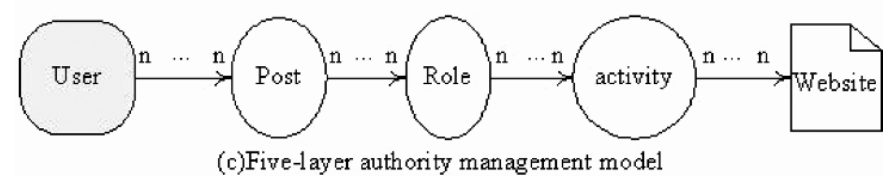

Fig. 1. Three kinds of authority management model

As for the problems existed in the model, $\mathrm{Yu}$ and Wang give a role network model (RNM) based on system theory and role theory (Yu Miao et al. 2003) (Fig. 1 (b)). Li etc. develop SODB-RNM based on the RNM mode (Li Huaiming et al. 2006). RNM model and SODB-RNM model extract the role deeply, and separate the role into two parts which named role and post. The authority change in the system would be complex when the user's post changes. Post, a role group, can solve the problem. SODB-RNM model is separated in several layers and applied to the system development and 
application, but its complexity makes the developing management be uneasy. Besides, RBAC, RNM and SODB-RNM is only limited to level of "activity" when they are applied to authority distribution management.

For solving the problems discussed above, this article put forward an authority management model whose systematic structure has five layers and two sections.

\section{FIVE LAYERS AND TWO SECTIONS AUTHORIZATION MANAGEMENT}

Inheriting the thought of RNM, the five layer and two sections model separates the role in RBAC model into post and role, in order to solve the authority management problems which could take place when the users' headship change. And it also expands the RNM and puts the website into the model for more accurate authority management. Based on the model, it is studied that the position of model's content in the system development. Personnel, post, role, activity and website (table) are combined to two systems to achieve unified and flexible authority management.

\subsection{Five layers authorization management}

In the RNM, the information and the access control function are based on the post and role: a user could have several posts and a post could have several roles; a role could handle several actions, an action could be handled by several roles. RNM could solve the big adjustment of authority, but both RNM and RBAC could not solve the problem that tables change in an action. In this article a five layer model is proposed to solve the problems that tables have changed in an action, for example, some tables do not need in an action, or adding a search of some tables, or adding a new table. This model also expands the RNM and introduces the website. As showing in Fig. 1(c), in five layers model, the action authority could be divided more specifically, and an action could handle several table (website), and a table also could be handled by different actions.

Fig. 2 shows kinds of situation when the authority of activity changes (the website of action handling changes). It can be seen that the model could adjust the system conveniently and promptly, so that it responds the service change in time. Without this model, the activities and tables would be coded stubbornly in the system; the system would be recompiled when tables change. The recompiling would not only increase the sustaining cost but also decrease the expansibility of the system. 


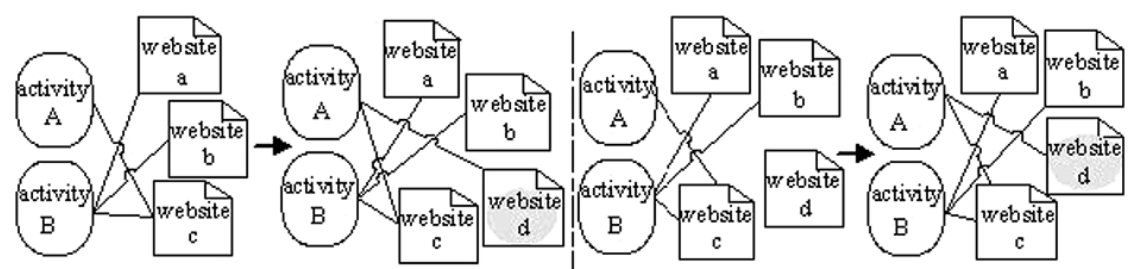

(a) add a new website

(c) comect a website to a activity

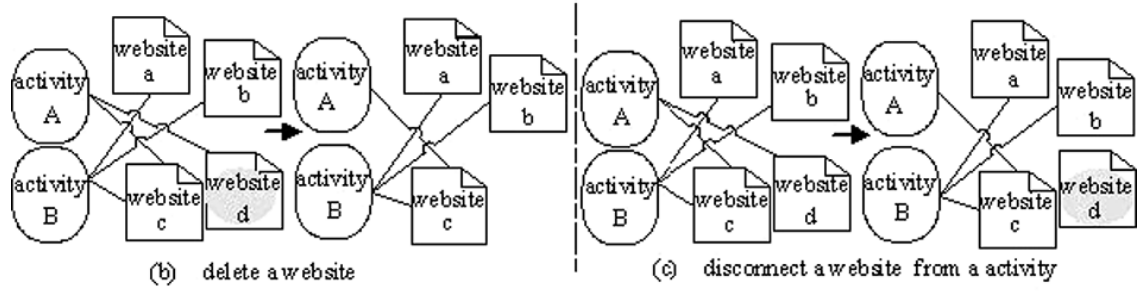

Fig. 2. The change of the activity authority

\subsection{Two sections authorization management}

Five Layers Authorization Management model can solve the problem of authority setup when user authority changed. However, it is difficult to decide the depth of common authority management, since every subsystem has its own authority managements. How to deal with the distribution of the authority management and how to harmonize the common user management subsystem is becoming a big problem. So a two sections authority management is developed, as Fig. 3 shows.

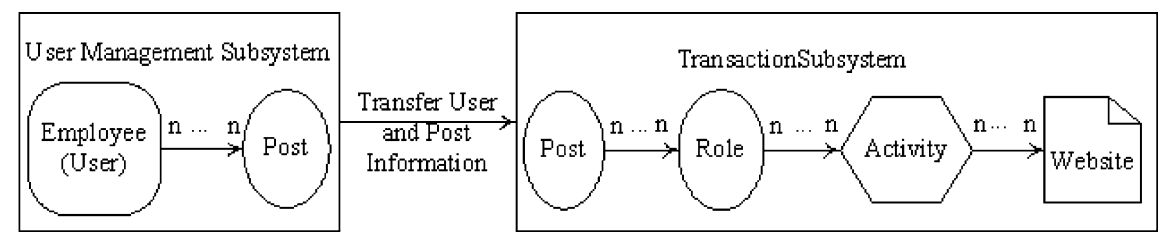

Fig. 3. Five layers and two sections authorization management model

In this "Two Sections Authorization Management", personnel and position management are extracted and are managed in subsystem of user management. The relations of position, role, website and activity are managed in specific operational system. In this way, the five layers model is separated into two sections:

The first section (rough adjustment): indicates administrators, positions and the relations between personnel and positions in subsystem of user management. Fig. 4(a) shows that the method is to read the information of user and post in the user management subsystem, instead of managing it. This method can solve the problem of internal position change; the change 
would have effect on all user authorities in all transaction subsystems, rather than on specific transactions. That's why it is called rough adjustment;

The second section (specific adjustment): post, roles, activities and the relations between them are managed in the detail operational system, as shown in Fig. 4(b). When the position authority changes or when the operation change results to role managing action change, what has to be changed are the relationsof positions and roles or relations of roles and activities, rather than subsystem of user management and other operational system. So this action cannot cause any influence to other operational systems. All these adjustments are related to authority position, so it can be called "specific adjustment".
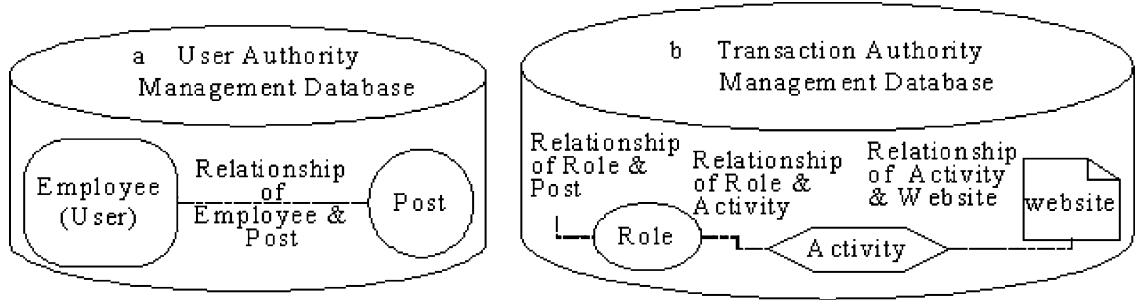

Fig. 4. Storage structure of user management subsystem \& other subsystems authority management

\subsection{The process of five layers and two sections authorization management}

As the Fig. 5 shows, assuming the authority exchanging between user $\mathrm{A}$ and $\mathrm{B}$, user $\mathrm{B}$ does not hold the role $\mathrm{b}$ and $\mathrm{B}$ is endued with a new position $\mathrm{A}$ which has role $\mathrm{c}$ in sub-system A, and he could log in the website 2. Take this situation for example to illustrate the process of Five Layers and Two Sections Authorization Management:

(1) Delete the relation between user B and post B, save the change in the user management database;

(2) Set up the relation between personnel B and post A, save the change in user management database;

(3) Read the information of personnel and post of application system A in user management system, then delete the relation between post $\mathrm{A}$ and role $\mathrm{b}$, save the change in the authority management database of the application system;

(4) Set up the relation between role $c$ and action $c$ in the application system A, save the change in the authority management database of the application system; 
(5) Set up the relation between the action c and website 2, save the change in the authority management database of the application system.

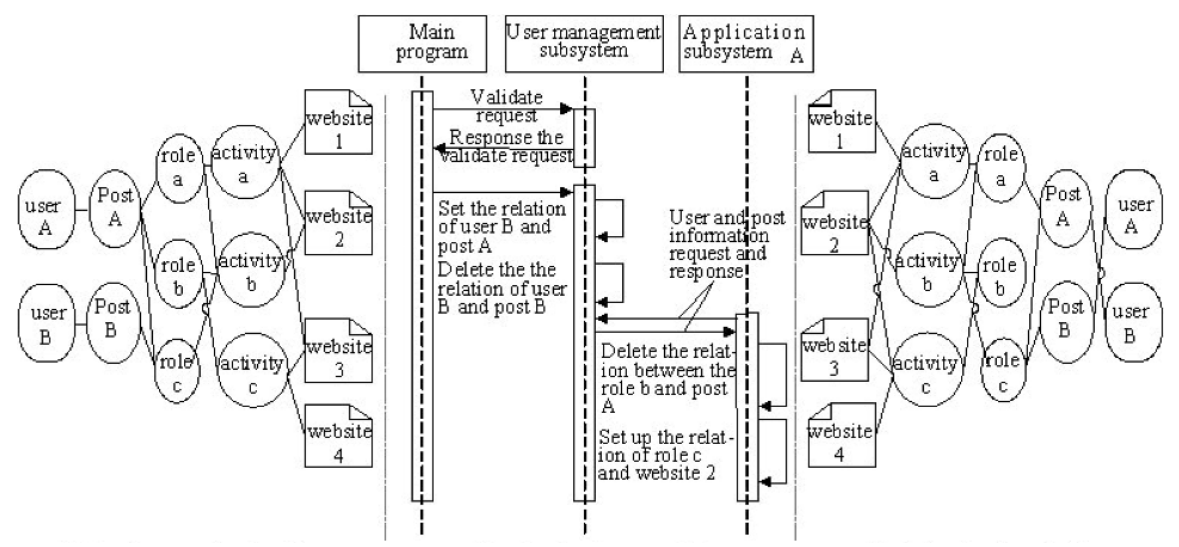

Fig. 5. The process of five layers and two sections authorization management model

From the adjustment process, it can be seen that Five Layers and Two Sections Authorization Management can consider not only the authority adjustment when user's positions have been little changed, but also the specific adjustment when user's authorities have been changed a lot. Therefore, the model is highly flexible so that it meets the requirement of authority management of one-stop office system.

\section{APPLICATION}

Five Layers and Two Sections Authorization Management model has been successfully applied to many constructions of Electronic Government's System, for example, the Land and Resources Information Management System (LRIMS) of Liuzhou city. The LRIMS contains 6 subsystems, including subsystem of cadastre management, land use and law supervision and so on. The whole system has adopted one-stop operation mode with receiving all kinds of business applications and distributing them to responsible department in the system. Because different operators use different subsystems and has different roles, the role management seems to be complicated. As the periodical internal positions adjustment of the Land Resource Bureau, there is a big change in the authority management mode base on roles. But if adopt the five layer and two section model, the workload will be decrease greatly. With the business development, when some tables or websites are changed, the advantage of flexibility and expansibility of the model could be seen more clearly. 


\section{CONCLUSION}

With the development of informatization, Electronic Government's System must be widely used in government offices. If the application complexity can be reduced, the one-stop office system must be widely used. One-stop office system combines several transaction subsystems together, which leads to the complexity of role management. Five Layers and Two Sections Authorization Management model separates authority management into five layers, and put rough adjustment and specific adjustment respectively in subsystem of user management and subsystem of specific transaction process. In this way, the problem of authority management complexity can be solved and workload can be reduced.

\section{ACKNOWLEDGEMENTS}

This study has been funded by China National 863 Plans Projects (Contract Number: 2006AA120102). This study has been applied to some systems programming, such as Liuzhou Land Resource Management Information System, Tangshan Digital Land Information System, and so on. Thank Liuzhou Land Resource Bureau, Tangshan Land Resource Bureau and other organizations for their data and support.

\section{REFERENCES}

Han Shengju, Ye Xin, Wang Yanzhang, Li Huaiming. 2006, Research on extended role network models of e-government application integration, Journal of Dalian University of Technology, Vol. 46, No. 2: 286-291.

Li Huaiming, Ye Xin, Wang Yanzhang. 2006, Organization and Empowerment Management of the Complex Government Affair Information System, Systems Engineering, Vol. 24, No. 4: 44-48.

Yan han, Zhang hong, Xu manwu. 2006, Organization and Empowerment Management of the Complex Government Affair Information System, System Engineering, 24, (4), 44-48.

Ye Xin, Li Huaiming, Wang Yanzhang. 2006, Research and Design of One-stop Administrative Permit System, Application Research of Computers, 2006, No. 4: 200-203.

Yu Miao, Wang Yanzhang. 2003, Research on an E-Gov Affairs System Architecture Based on Role Network Model \& Its Realization. Computer Engineering and Applications. Vol. 39, No. 12: 31-35. 